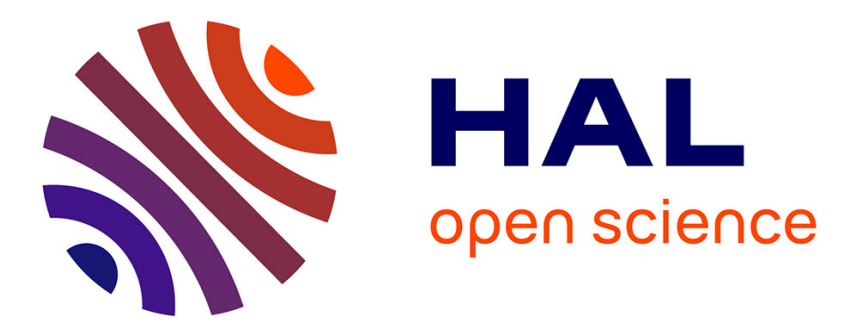

\title{
Toward an effective strategy in glioblastoma treatment. Part II: RNA interference as a promising way to sensitize glioblastomas to temozolomide.
} Khaled Messaoudi, Anne Clavreul, Frédéric Lagarce

\section{- To cite this version:}

Khaled Messaoudi, Anne Clavreul, Frédéric Lagarce. Toward an effective strategy in glioblastoma treatment. Part II: RNA interference as a promising way to sensitize glioblastomas to temozolomide.. Drug Discovery Today, 2015, 20 (6), pp.772-9. 10.1016/j.drudis.2015.02.014 hal-01392444

HAL Id: hal-01392444

https://hal.science/hal-01392444

Submitted on 20 Sep 2021

HAL is a multi-disciplinary open access archive for the deposit and dissemination of scientific research documents, whether they are published or not. The documents may come from teaching and research institutions in France or abroad, or from public or private research centers.
L'archive ouverte pluridisciplinaire HAL, est destinée au dépôt et à la diffusion de documents scientifiques de niveau recherche, publiés ou non, émanant des établissements d'enseignement et de recherche français ou étrangers, des laboratoires publics ou privés.

\section{(c)(1)}

Distributed under a Creative Commons Attribution| 4.0 International License 


\title{
Toward an effective strategy in glioblastoma treatment. Part II: RNA interference as a promising way to sensitize glioblastomas to temozolomide
}

\author{
Khaled Messaoudi ${ }^{1,2}$, Anne Clavreul ${ }^{1,2}$ and Frédéric Lagarce ${ }^{1,2,3}$ \\ ${ }^{1}$ LUNAM Université, Angers, France \\ ${ }^{2}$ Inserm U1066, Micro et Nanomédicines Biomimétiques, IBS, Angers Cedex 9, France \\ ${ }^{3}$ Service Pharmacie, CHU Angers, France
}

RNA interference (RNAi) is a strategy of gene regulation that has opened up many opportunities for the treatment of cancers, especially glioblastoma multiforme (GBM). This strategy reduced the expression of many proteins involved in the resistance of these tumors to anticancer drugs, particularly to temozolomide (TMZ). A significant research effort has gone into RNAi delivery and target selection for clinical application of this new discovery in the treatment of GBMs. However, some limitations must be resolved to enhance the safety of RNAi-based therapeutics and to reduce their immune response. In this review, the mechanism of RNAi will be described. Moreover, the opportunities offered by RNAi strategy to reverse the phenotype of these tumor cells as well as prospects and challenges ahead in the RNAi-based therapy will be discussed.

\section{Introduction}

Resistance to anticancer drugs is a problem in many cancers, particularly glioblastoma multiforme (GBM) - the most common type of primary brain tumor [1]. The prognosis of these tumors remains poor with a median survival of 14.6 months despite receiving many therapies including surgery, radiotherapy and chemotherapy [2]. Combining radiation therapy with temozolomide (TMZ) is currently the first-line therapy for GBMs. However, the efficiency of TMZ remains limited owing to inherent and acquired resistance of glial tumor cells. The main resistance mechanisms have been detailed in part I of this review series. Different strategies of inhibiting the effect of these proteins involved in resistance to TMZ were tested. However, the effects obtained are often inadequate or disappointing.

Development of the RNA interference (RNAi) strategy has opened new perspectives for the treatment of these malignancies. Indeed, the principle of RNAi is based on the reduction of expression of a target mRNA into protein. This strategy has been applied with success in vitro and in vivo in different pathologies including cancers, viral infections and metabolic disorders [3]. However, transport and delivery of interfering RNA requires the use of

Corresponding author: Lagarce, F. (frederic.lagarce@univ-angers.fr) vectors that must be safe and efficient. In this review, we will initially describe the RNAi strategy mechanism, followed by a description of different nonviral vectors used for the transport and delivery of interfering RNA. Finally, application of this strategy in GBMs and the various challenges and obstacles that must be overcome for successful clinical application will be discussed.

\section{RNAi mechanism in mammalian cells}

In recent years, the strategy of RNAi, the principle of which is based on inhibition of protein synthesis by targeting a specific mRNA, was discovered and has opened up new perspectives in human disease treatment. RNAi is an intrinsically cellular pathway that was discovered in 1998 [4,5]. Two classes of small RNAs have been identified to mediate RNAi, small interfering RNA (siRNA) and micro RNA (miRNA).

For siRNA-mediated RNAi, the cellular process begins with long double-stranded RNAs (dsRNAs) cleaved in the cytoplasm by the enzyme Dicer to generate mature siRNAs of about 21-23 base pairs (bp). The resulting siRNAs are incorporated into RNA-induced silencing complex (RISC), which becomes active. Then, the antisense strand remains in the RISC complex and guides the RNAi enzymatic machinery while the sense strand is eliminated from the RISC complex. The RISC-containing guide strand binds to 


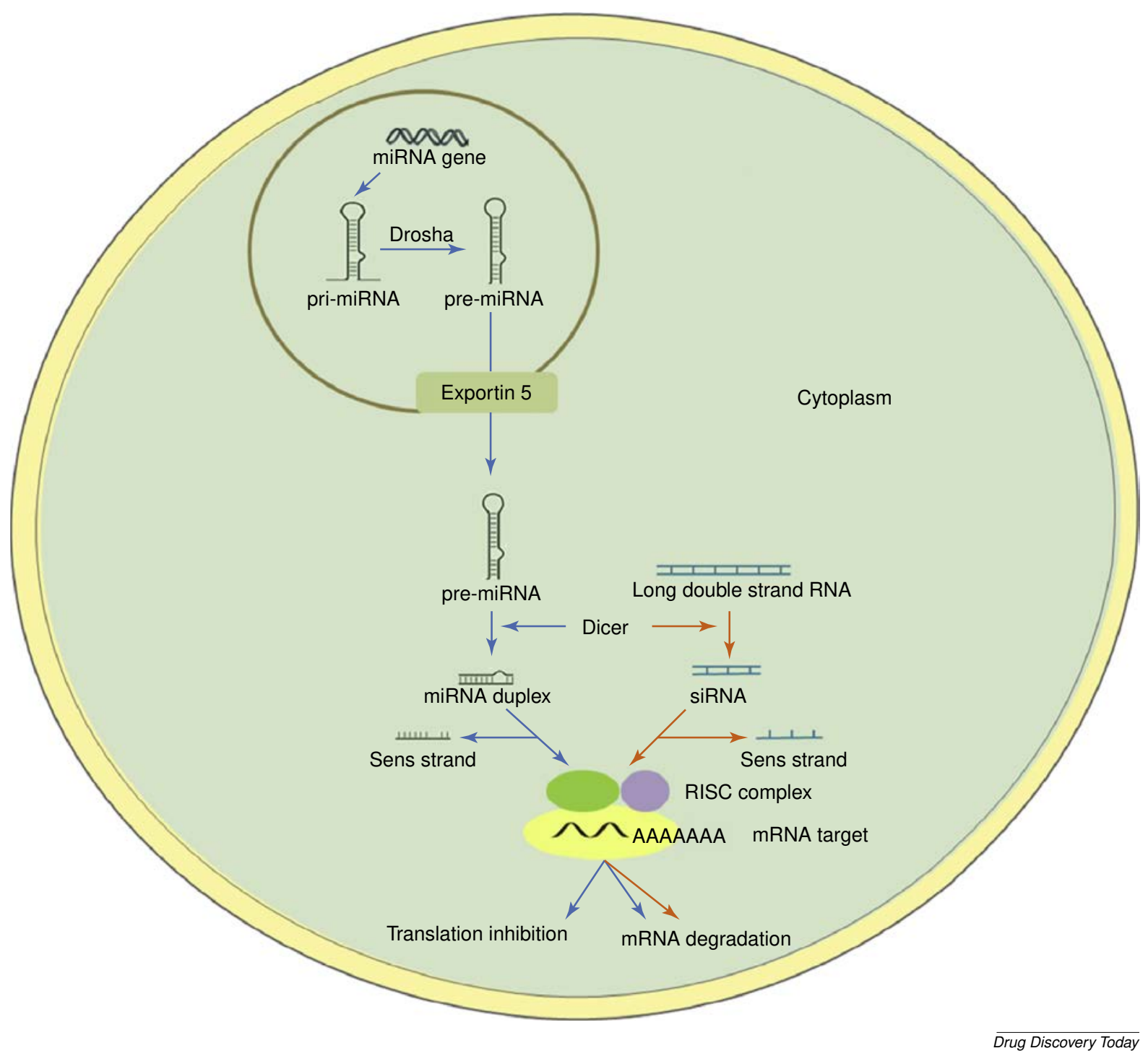

FIGURE 1

Mechanism of action of RNA interference (RNAi) by silencing RNA (siRNA) or micro RNA (miRNA) in mammalian cells.

complementary mRNA, which causes its degradation by the enzyme argonaute 2 (Ago-2) [6,7] (Fig. 1).

Concerning miRNA-mediated RNAi, genes encoding miRNAs are transcribed into long primary transcripts (pri-miRNAs) that are cleaved by RNAse III Drosha to generate precursor miRNAs (premiRNAs) in the nucleus [8,9]. Pre-miRNAs are transported from the nucleus to the cytoplasm by exportin-5 and then processed by RNAse III Dicer to generate mature miRNAs of about 22 nucleotides in length [8]. Then, miRNAs are incorporated into the RISC complex and bind through imperfect complementarity to the $3^{\prime}$ untranslated region (UTR) of its mRNA target, leading to translational repression or mRNA degradation $[8,10]$. A single miRNA molecule is probably capable of regulating multiple mRNAs, and conversely one mRNA can be regulated by multiple miRNAs (Fig. 1).

\section{RNAi delivery}

The use of these small RNA molecules alone as therapeutics is not possible for several reasons. Among these is their negative charge which prevents them from crossing the plasma membrane to reach their target in the cytoplasm $[6,11]$. The presence of nucleases in plasma and in the cytoplasm are also likely to deteriorate these biopolymers rapidly after injection [12], not to mention the immune response that can be triggered by their presence in the blood [12]. These reasons emphasize the need for a suitable vector able to deliver these small RNA molecules into the desired cells.

Viral and nonviral vectors were developed to carry siRNAs to their site of action located in the cytoplasm [13]. The RNAi induced by viral delivery was demonstrated using different viruses particularly adeno-associated virus (AAV), herpes simplex virus (HSV) and lentiviral vectors $[8,14]$. Despite their efficiency, viral vectors have some limitations such as their residual pathogenic effect, which represents a potential risk to patients [15]. Moreover, a mutagenesis effect was observed in some clinical trials using these vectors. This is why a lot of research has been focused on the development of nonviral vectors. An ideal nonviral vector has certain attributes that are: (i) to be biocompatible and biodegradable; (ii) to be able to protect nucleic acids against nuclease degradation in plasma and avoid their renal clearance; and (iii) 
to allow a reversible binding of nucleic acids and to trigger their release at the site of cellular action.

Different carriers of siRNAs and miRNAs were developed and described in the literature; most of them are positively charged to allow attachment of nucleic acids that are negatively charged and to enable a better interaction with the cell membrane and improve the cellular uptake [16]. Among these vectors, there are polymeric nanoparticles, lipid-based systems and inorganic nanoparticles.

\section{Polymeric nanoparticles}

Cationic polymers can interact with the negatively charged phosphate groups of nucleic acids, which create complexes called polyplexes. Natural and synthetic polymers are used for RNAi delivery in mammalian cells [17].

Polyethyleneimine. Polyethyleneimine (PEI) is a synthetic polymer considered to be the most effective polymer for siRNA delivery. The high density of positive charge provided by this polymer creates strong links with nucleic acids, thereby protecting them from enzymatic degradation by nucleases. Factors such as molecular weight (MW) and degree of branching of PEI affect the efficiency and the toxicity of the polymer [18]. Indeed, the high MW chains are more effective for siRNA delivery compared with the low MW chains. Similarly, branched PEI is more efficient for siRNA binding than the linear form. However, the high MW chains and branched chains are the most toxic [18] (Fig. 2).

PEI-siRNA complexes are internalized by endocytosis in various cells. This pathway is composed of vesicles known as endosomes (with an internal $\mathrm{pH}$ of approximately $\mathrm{pH}$ 5) that mature from early endosomes to late endosomes before fusing with lysosomes, which contain digestive enzymes [19]. The acidic lysosomal environment causes the protonation of the amine groups of PEI that, owing to the proton sponge effect as a result of its high buffering capacity, induces osmotic swelling of the endocytosis vesicle until vesicle rupture, leading to the release of polyplexes into the cytoplasm [20]. The ability of PEI to escape lysozomal degradation enables the release of intact siRNA and miRNA in the cytoplasm and thus provides a high efficiency of gene silencing [19-21].

Chitosan. Chitosan is another polymer used for RNAi delivery. This natural polysaccharide consists of repeating D-glucosamine and $N$-acetyl D-glucosamine units linked by $\beta(1-4)$ glycosidic bonds [22]. It is obtained by deacetylation of chitin and is considered to be a nontoxic and biocompatible polymer [23].

Chitosan MW, degree of deacetylation (DDA) and N:P ratio (ratio of the protonated amine groups of chitosan on the phosphate groups of the siRNA) are factors that greatly influence siRNA transfection efficiency [24]. Indeed, higher MW chains are flexible allowing them to wrap the siRNAs in stable complexes and protect them from nuclease degradation. However, this stability can be an obstacle to the release of siRNAs in the cytoplasm. In addition, high MW chitosan showed drawbacks such as aggregation and low solubility at neutral $\mathrm{pH}$. Low MW chains enables more-efficient intracellular release but low complexation [24]. Higher DDA enhances electrostatic interaction with siRNAs, which leads to a greater stability of the complexes formed with siRNAs [25] (Fig. 2). The excess of positive charges in comparison with the negative charges increases the stability of the chitosan-siRNA complexes and increases uptake across anionic cell surface [25].

Dendrimers. Dendrimers are synthetic macromolecules; their structure is composed of a central core, branches of repeating units and terminal groups, which can interact with charged molecules such as nucleic acids, imaging agents and anticancer drugs $[26,27]$. In addition, the internal hydrophobic cavities of dendrimers can encapsulate hydrophobic anticancer drugs [28] (Fig. 2).

Dendrimers with positively charged surface groups are used for RNAi delivery $[29,30]$. Poly (amidoamine) dendrimers (PAMAM) are the most important type in the dendrimer family [31]. PAMAM possess tertiary amine functions in their structure, which can be protonated in acidic medium and thus confer a proton sponge effect [32], which allows a release of siRNA and miRNA on the cell level while preventing degradation in the endolysosomal compartment $[31,33]$. Some studies showed that PAMAM dendrimers are excellent nonviral vectors for siRNA and miRNA delivery, thereby producing powerful gene silencing in vitro and in vivo [34-36]. Despite their transfection efficiency, cationic and higher generation dendrimers have the disadvantage of interacting with blood components, destabilizing cell membranes and causing cell lysis [37].

\section{Lipid-based systems}

Liposomes. Liposomes are efficient vectors for the delivery of drugs and nucleic acids [38]. Liposomal forms of doxorubicin

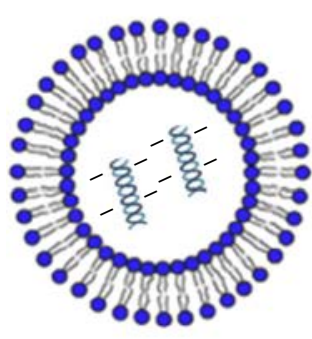

Liposome

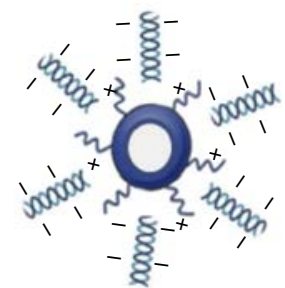

Lipid nanocapsules

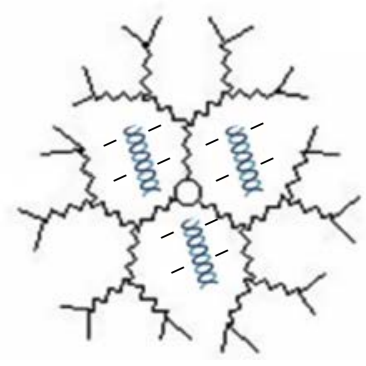

Dendrimers

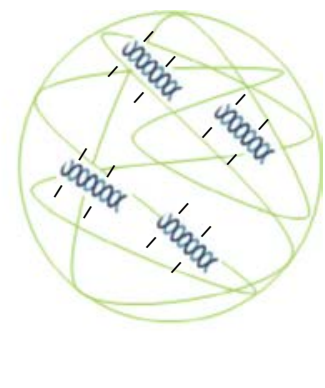

Polymeric nanoparticles $\overline{\text { Drug Discovery Today }}$

FIGURE 2

Different types of nanocarriers for delivery of silencing RNA (siRNA) used for RNA interference. Negatively charged siRNA interact with positive charges of the nanocarrier components. 
and amphotericin B obtained FDA approval for the treatment of Kaposi's sarcoma and fungal infections, respectively [39]. Liposomes are formed by unilamelar or multilamelar phospholipid bilayers. Three classes of phospholipids are used in the formulation of liposomes: anionic, cationic and neutral phospholipids [17,39] (Fig. 2).

Owing to their hydrophilic nature, the nucleic acids can be encapsulated in the core of liposomes. Conversely, cationic lipids are the most efficient for the transport of nucleic acids, because they can interact as cationic polymers that create lipoplexes $[20,40]$. In addition to their ability to encapsulate drugs, the liposome surface can be functionalized to allow, firstly, an avoidance of recognition by the immune system (i.e. pegylated stealth liposomes) increasing the plasma half-life [41] and, secondly, to target tumor cells specifically through the use of various ligands such as specific antibodies [42]. Despite their transfection efficiency of nucleic acids, cationic liposomes have the drawback of being toxic by their interaction with negatively charged cellular components (i.e. opsonins, serum protein and enzymes) resulting in hemolysis and activation of the complement system, which causes their rapid elimination [43].

Lipid nanoparticles. Various types of lipid nanoparticles have been developed. Among them, the lipid nanocapsules (LNCs) have a structure that is a hybrid between polymeric nanocapsules and liposomes because of their oily core, which is surrounded by a membrane made of tensioactive agents such as PEG hydroxystearate $\left(\right.$ Solutol $\left.{ }^{\mathbb{R}}\right)$. LNCs have many advantages such as increased safety, high stability and the possibility of lipophilic drug encapsulation (e.g. paclitaxel) [44]. They can be produced without organic solvents by a phase inversion process and with generally recognized as safe (GRAS) excipients and are also genetically modified organism (GMO)-free [45]. The concept of this process uses the specific ability of some polyethoxylated surfactants to modify their affinities for water and oil as a function of the temperature [46]. These nanocapsules have shown their efficiency for the delivery of nucleic acids. Indeed, siRNA can be entrapped into LNCs after formation of lipoplexes with cationic lipids [47]. Another strategy consists of surface modification of LNCs by grafting a cationic polymer such as chitosan to obtain a cationic vector capable of fixing nucleic acids by electrostatic attraction [48] (Fig. 2).

\section{Inorganic nanoparticles}

A number of inorganic nanoparticles such as carbon nanotubes, magnetic nanoparticles and gold nanoparticles have been developed for gene therapy [49]. These systems are different from one another because of their composition and the methods of formulation used.

Carbon nanotubes. Some studies have shown that carbon nanotubes easily cross the plasma membrane using an endocytosisindependent mechanism [49]. Several functionalized carbon nanotubes have been designed and tested for siRNA and miRNA delivery [50-53]. The most effective formulations of carbon nanotubes were functionalized with amino groups [54]. Despite their efficiency in the transport of nucleic acids, their toxicity profile caused problems for their use in the clinic $[55,56]$.

Magnetic nanoparticles. Magnetic nanoparticles were developed for tumor imaging and drug delivery. siRNA delivery was tested with different magnetic nanoparticles, such as iron oxide nanoparticles, and has shown promising results [49]. The advantage of these nanoparticles is the possibility to deliver siRNA to the desired tissue by applying an external magnetic field [57].

Gold nanoparticles. Gold nanoparticles are also used as nonviral vectors and present several opportunities for RNAi delivery because of their interesting properties, in particular their biocompatibility and low toxicity $[58,59]$. Their stability enables avoiding interactions with serum ionic compounds in particular proteins [60]. Gold nanoparticles possess photothermal properties that can be exploited for cancer therapy [60]. In addition, their surface can be functionalized with specific ligands to allow easy fixation of small RNA molecules for gene therapy $[60,61]$.

\section{RNAi delivery system used to sensitize GBM cells to TMZ}

Studies that used RNAi to sensitize GBM cells to TMZ are summarized in Table 1 . These results show that different targets are to be reached by siRNA for the treatment of GBMs. The administration of RNAi can be done directly into the tumor or intravenously. The results obtained in these studies are very promising. Despite this, only a few nonviral vectors are approved by the FDA and are in clinical trials, demonstrating that there are still some barriers to overcome, particularly in terms of selectivity, efficacy and toxicity.

Among the vectors that have reached clinical trials, the Ron$\mathrm{del}^{\mathrm{TM}}$ system produced by Calando Pharmaceuticals was used in clinical trial CALAA-01 indicated for the treatment of cancers and solid tumors refractory to conventional therapy. It used an siRNA targeting the M2 subunit of ribonuclease reductase, a key enzyme in the synthesis and replication of DNA that converts ribonucleotides into deoxyribonucleotides. Rondel ${ }^{\mathrm{TM}}$ vector consists of alternating $\beta$-cyclodextrins and a positively charged polymer to allow attachment of siRNA by electrostatic attraction; the formed nanocomplexes have a diameter of less than $100 \mathrm{~nm}$. Each cyclodextrin molecule contains in its hydrophobic cavity a molecule of admantane, which is covalently bound to one molecule of polyethylene glycol (PEG). The other end of the PEG molecule is linked to a transferrin molecule that will allow the nanocarrier to bind to the transferrin receptor (TfR) overexpressed in tumor cells [62].

For clinical application, RNAi-based cancer therapeutics should specifically recognize cancer cells without affecting normal cells. The selectivity of nanocarriers was improved by their conjugation to specific ligands that can bind the tumor cell receptors. However, to reach their target in cancer cells, it is necessary to increase the circulation time of these particles in plasma by protecting them against a capture by the reticuloendothelial cell system (RES). This protection can be efficiently achieved through a PEG coating that also has the drawback of reducing the tumor cell uptake of these particles [49].

It is crucial to improve vector safety because even though many vectors have proven their efficiency in the transport and delivery of drugs and nucleic acids they often cause a stimulation of the immune response and cytotoxicity that does not allow their clinical use. The safety profile of vectors could be improved through the use of biocompatible, biodegradable and nontoxic compounds [49]. In addition to efficacy, selectivity and safety, vectors must be produced on a large scale to allow their use in clinical trials. However, the production of nonviral vectors at a 


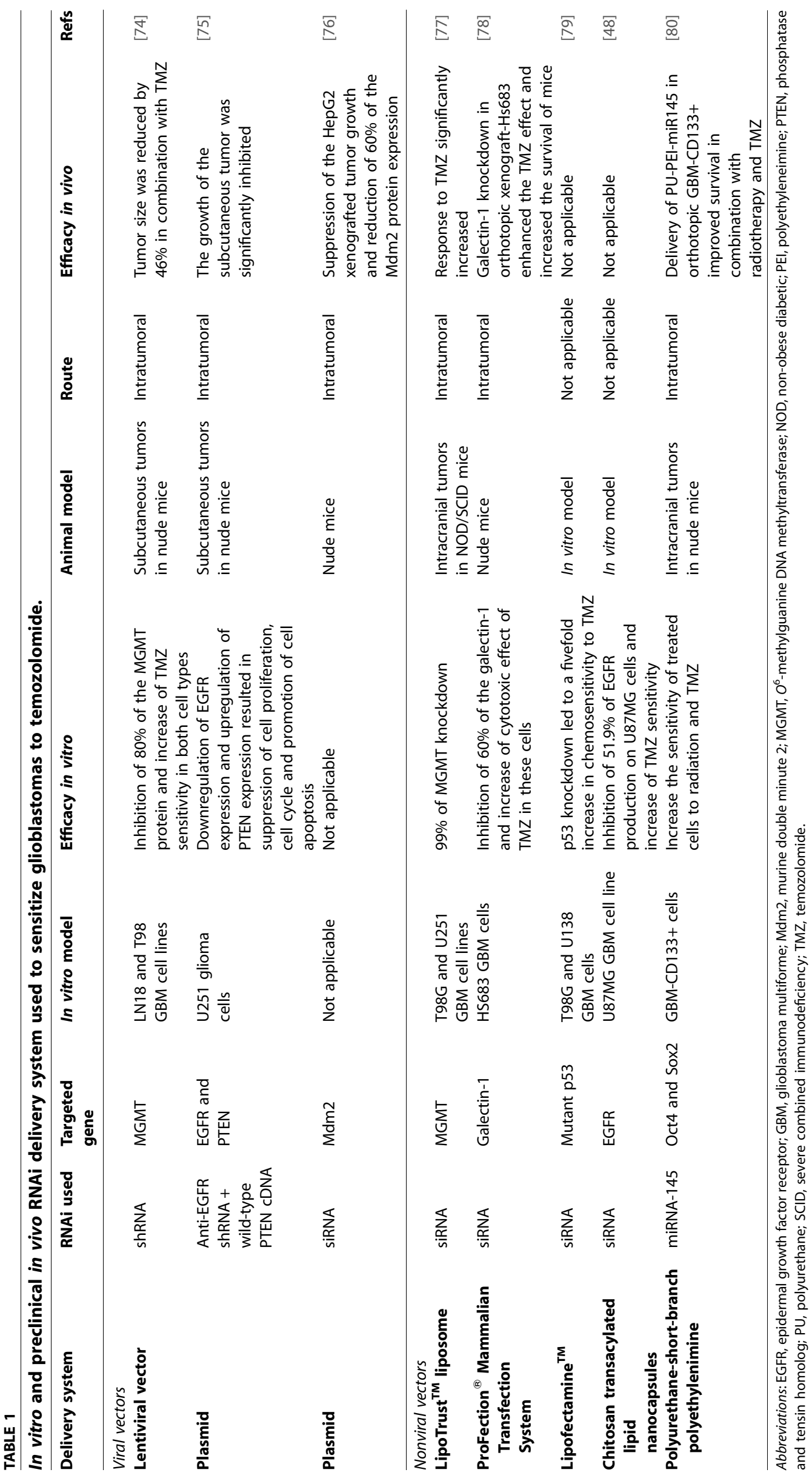


large scale has only been demonstrated with a limited number of drug delivery systems, this impedes the use of these promising formulations in clinical trials [63].

One limitation to the use of siRNA in the clinics is the off-target effect due to the imperfect complementarity between the siRNA and the $3^{\prime}$ UTR of the mRNA transcripts. This leads to the inhibition of genes other than the target genes that might have important consequences for cell function [64]. To reduce or eliminate this effect, chemical modifications of the nucleic acids averted complementarity with other mRNAs other than those targeted $[64,65]$. The short in vivo effect of siRNA (about one week) is another limitation for their use that requires repeated administration and causes a massive accumulation of vectors and siRNA into tumors responsible for toxicity [66]. It is thus necessary to obtain the most biocompatible vectors to prevent their toxic effect.

The saturation of the cellular enzymatic machinery by exogenous interfering RNA is another problem that can cause disruption of cellular functions controlled by small RNAs such as endogenous miRNA. Indeed, exportin-5 is a key protein of the miRNA pathway that can be saturated with exogenous miRNA $[64,67]$. By contrast, competition is also present for the incorporation of interfering RNA in the RISC complex. This competition is problematic because the endogenous miRNA present incorporation kinetics slower than that of exogenous RNA [68].

For GBM treatment using the intravenous route, an siRNA carrier must cross the blood-brain barrier (BBB) to release the interfering RNA. This barrier protects the brain from toxins and drugs presented in the circulation; this protection is ensured in particular by the tight junctions and efflux pumps such as Pglycoprotein (P-gp) that actively remove these molecules from the circulation, which explains the failure of some chemotherapy [69]. Although this barrier seems to be somewhat disrupted in the abnormal vascular networks characterizing GBMs, it frequently remains intact along the infiltrating area where the plurality of recurrences tend to occur [70].

By contrast, the local administration of nanocarriers avoids the crossing of the BBB and allows drug delivery directly into the brain parenchyma. The major drawback of this technique is a poor diffusion of particles in the tumor, which does not allow the drug to reach the area of the recurrence of GBMs located a few centimeters from the original tumor area [71]. Moreover, particle reflux from the tumor area is often encountered which reduces the effectiveness of treatment. This is partly solved by the development of convectionenhanced delivery (CED), which allows injecting the particles under a pressure gradient for more-homogeneous distribution in the tumor [72]. With this technique, the drug concentrations in brain tumors can be superior to those obtained with systemic administration.

In addition, GBMs are very heterogeneous tumors characterized by the parallel overexpression of multiple proteins that are involved in resistance to TMZ. This implies that, for effective treatment by RNAi, it is necessary to adapt the therapy to each patient based on their molecular characteristics. Also, GBM resistance to TMZ can be innate or acquired, which can cause a rapid recurrence of tumor after initial remission. This illustrates that the success of a therapy requires knowledge of the gene expression profile of the tumor at different times to adapt the treatment with siRNA and possibly target other proteins involved in this new induced resistance [70]. Despite its effectiveness, drug administration using CED has several disadvantages including poor distribution distance of the drug to the site of placement of the catheter $(<3 \mathrm{~cm})$ which is a problem knowing the infiltrating and invasive character of GBMs [73]. The complexity of brain tumors associated with edema and leakage of drugs into the subarachnoid space are drawbacks that will have to be addressed for a better use of this technique [73].

\section{Concluding remarks}

Concomitant administration of TMZ and radiotherapy improved prognosis of patients with GBM by increasing median survival, but this effect remains modest. The development of molecular biology has enabled the understanding of the involvement of certain genes in cancer resistance and allowed the molecular profile of each tumor to be defined. RNAi is one of the most promising strategies to overcome cancer resistance. Indeed, this strategy can be applied to achieve different targets involved in GBM resistance to TMZ. These targets could be identified by genetic screening to find the genes that are out of control for every individual. This concept known as personalized medicine adapts the therapeutic strategy for each patient, which is a major advantage given the heterogeneity of GBMs. In addition, the development of nanocarriers able to transport drugs and nucleic acids provides a synergistic effect with lower effective doses of these drugs and fewer side-effects. Further investigations are still being carried out to improve the safety of these nanocarriers and their effectiveness for clinical application.

\section{Acknowledgments}

The authors are very grateful to the Ligue contre le Cancer, Comité du Maine et Loire, which founded this work.

\footnotetext{
References

1 Stupp, R. et al. (2005) Radiotherapy plus concomitant and adjuvant temozolomide for glioblastoma. N. Engl. J. Med. 352, 987-996

2 Lefranc, F. et al. (2006) Present and potential future issues in glioblastoma treatment. Expert Rev. Anticancer Ther. 6, 719-732

3 Williford, J.M. et al. (2014) Recent advances in nanoparticle-mediated siRNA delivery. Annu. Rev. Biomed. Eng. 16, 347-370

4 Fire, A. et al. (1998) Potent and specific genetic interference by double-stranded RNA in Caenorhabditis elegans. Nature 391, 806-811

5 McManus, M.T. and Sharp, P.A. (2002) Gene silencing in mammals by small interfering RNAs. Nat. Rev. Genet. 3, 737-747

6 Aagaard, L. and Rossi, J.J. (2007) RNAi therapeutics: principles, prospects and challenges. Adv. Drug Deliv. Rev. 59, 75-86
}

\footnotetext{
7 Kim, D.H. and Rossi, J.J. (2007) Strategies for silencing human disease using RNA interference. Nat. Rev. Genet. 8, 173-184

8 Guo, D. et al. (2010) RNA interference therapy for glioblastoma. Expert Opin. Biol. Ther. 10, 927-936

9 Lee, Y. et al. (2003) The nuclear RNase III Drosha initiates microRNA processing. Nature 425, 415-419

10 Peterson, S.M. et al. (2014) Common features of microRNA target prediction tools. Front. Genet. 5, 23

11 Reischl, D. and Zimmer, A. (2009) Drug delivery of siRNA therapeutics: potentials and limits of nanosystems. Nanomedicine 5, 8-20

12 Volkov, A.A. et al. (2009) Selective protection of nuclease-sensitive sites in siRNA prolongs silencing effect. Oligonucleotides 19, 191-202
} 
13 David, S. et al. (2010) Non-viral nanosystems for systemic siRNA delivery. Pharmacol. Res. 62, 100-114

14 Kota, J. et al. (2009) Therapeutic microRNA delivery suppresses tumorigenesis in a murine liver cancer model. Cell 137, 1005-1017

15 Hacein-Bey-Abina, S. et al. (2008) Insertional oncogenesis in 4 patients after retrovirus-mediated gene therapy of SCID-X1. J. Clin. Invest. 118, 3132-3142

$16 \mathrm{Kim}$, S.S. et al. (2009) Strategies for targeted nonviral delivery of siRNAs in vivo. Trends Mol. Med. 15, 491-500

17 Nikitenko, N.A. and Prassolov, V.S. (2013) Non-viral delivery and therapeutic application of small interfering RNAs. Acta Naturae 5, 35-53

18 Lungwitz, U. et al. (2005) Polyethylenimine-based non-viral gene delivery systems. Eur. J. Pharm. Biopharm. 60, 247-266

19 Varkouhi, A.K. et al. (2011) Endosomal escape pathways for delivery of biologicals. J. Control. Release 151, 220-228

20 Zhou, J. et al. (2013) Nanoparticle-based delivery of RNAi therapeutics: progress and challenges. Pharmaceuticals 6, 85-107

21 Ibrahim, A.F. et al. (2011) MicroRNA replacement therapy for miR-145 and miR-33a is efficacious in a model of colon carcinoma. Cancer Res. 71, 5214-5224

22 Buschmann, M.D. et al. (2013) Chitosans for delivery of nucleic acids. Adv. Drug Deliv. Rev. 65, 1234-1270

23 Mao, S. et al. (2010) Chitosan-based formulations for delivery of DNA and siRNA. Adv. Drug Deliv. Rev. 62, 12-27

24 Jin, L. et al. (2014) Current progress in gene delivery technology based on chemical methods and nano-carriers. Theranostics 4, 240-255

$25 \mathrm{Liu}$, X. et al. (2007) The influence of polymeric properties on chitosan/siRNA nanoparticle formulation and gene silencing. Biomaterials 28, 1280-1288

26 Biswas, S. and Torchilin, V.P. (2013) Dendrimers for siRNA delivery. Pharmaceuticals 6, 161-183

27 Menjoge, A.R. et al. (2010) Dendrimer-based drug and imaging conjugates: design considerations for nanomedical applications. Drug Discov. Today 15, 171-185

28 Zhang, M. et al. (2011) Multifunctional dendrimer/combretastatin A4 inclusion complexes enable in vitro targeted cancer therapy. Int. J. Nanomed. 6, 2337-2349

29 Perez, A.P. et al. (2009) Ethylendiamine core PAMAM dendrimers/siRNA complexes as in vitro silencing agents. Int. J. Pharm. 380, 189-200

30 Taratula, O. et al. (2009) Surface-engineered targeted PPI dendrimer for efficient intracellular and intratumoral siRNA delivery. J. Control. Release 140, 284-293

$31 \mathrm{Wu}$, J. et al. (2013) Dendrimers as carriers for siRNA delivery and gene silencing: a review. Scientific World J. 2013, 630654

32 Kukowska-Latallo, J.F. et al. (1996) Efficient transfer of genetic material into mammalian cells using Starburst polyamidoamine dendrimers. Proc. Natl. Acad. Sci. U. S. A. 93, 4897-4902

33 Zhang, Y. et al. (2013) Progress in microRNA delivery. J. Control. Release 172, 962 974

34 Liu, X. et al. (2013) Poly(amido amine) is an ideal carrier of miR-7 for enhancing gene silencing effects on the EGFR pathway in U251 glioma cells. Oncol. Rep. 29, 1387-1394

35 Liu, X. et al. (2014) Structurally flexible triethanolamine-core poly(amidoamine) dendrimers as effective nanovectors to deliver RNAi-based therapeutics. Biotechnol. Adv. 32, 844-852

36 Liu, X. et al. (2012) Efficient delivery of sticky siRNA and potent gene silencing in a prostate cancer model using a generation 5 triethanolamine-core PAMAM dendrimer. Mol. Pharm. 9, 470-481

37 Aillon, K.L. et al. (2009) Effects of nanomaterial physicochemical properties on in vivo toxicity. Adv. Drug Deliv. Rev. 61, 457-466

38 Akhtar, S. and Benter, I.F. (2007) Nonviral delivery of synthetic siRNAs in vivo. J. Clin. Invest. 117, 3623-3632

39 Ozpolat, B. et al. (2014) Liposomal siRNA nanocarriers for cancer therapy. Adv. Drug Deliv. Rev. 66, 110-116

40 Rai, K. et al. (2011) Liposomal delivery of MicroRNA-7-expressing plasmid overcomes epidermal growth factor receptor tyrosine kinase inhibitor-resistance in lung cancer cells. Mol. Cancer Ther. 10, 1720-1727

41 Pereira, D.M. et al. (2013) Delivering the promise of miRNA cancer therapeutics. Drug Discov. Today 18, 282-289

42 Zhang, Y. et al. (2004) Intravenous RNA interference gene therapy targeting the human epidermal growth factor receptor prolongs survival in intracranial brain cancer. Clin. Cancer Res. 10, 3667-3677

43 Zhang, J. et al. (2014) Non-viral nanocarriers for siRNA delivery in breast cancer. J. Control. Release 190C, 440-450

44 Hureaux, J. et al. (2010) Toxicological study and efficacy of blank and paclitaxelloaded lipid nanocapsules after i.v. administration in mice. Pharm. Res. 27, $421-430$
45 Hureaux, J. et al. (2009) Lipid nanocapsules: ready-to-use nanovectors for the aerosol delivery of paclitaxel. Eur. J. Pharm. Biopharm. 73, 239-246

46 Heurtault, B. et al. (2002) A novel phase inversion-based process for the preparation of lipid nanocarriers. Pharm. Res. 19, 875-880

47 Resnier, P. et al. (2013) EGFR siRNA lipid nanocapsules efficiently transfect glioma cells in vitro. Int. J. Pharm. 454, 748-755

48 Messaoudi, K. et al. (2014) Anti-epidermal growth factor receptor siRNA carried by chitosan-transacylated lipid nanocapsules increases sensitivity of glioblastoma cells to temozolomide. Int. J. Nanomed. 9, 1479-1490

49 Lee, J.M. et al. (2013) Recent developments in nanoparticle-based siRNA delivery for cancer therapy. Biomed. Res. Int. 2013, 782041

50 Bates, K. and Kostarelos, K. (2013) Carbon nanotubes as vectors for gene therapy: past achievements, present challenges and future goals. Adv. Drug Deliv. Rev. 65, 2023-2033

51 Wang, X. et al. (2008) Targeted RNA interference of cyclin A2 mediated by functionalized single-walled carbon nanotubes induces proliferation arrest and apoptosis in chronic myelogenous leukemia K562 cells. ChemMedChem 3, 940-945

52 Chen, H. et al. (2012) Functionalization of single-walled carbon nanotubes enables efficient intracellular delivery of siRNA targeting MDM2 to inhibit breast cancer cells growth. Biomed. Pharmacother. 66, 334-338

53 Kam, N.W. et al. (2005) Functionalization of carbon nanotubes via cleavable disulfide bonds for efficient intracellular delivery of siRNA and potent gene silencing. J. Am. Chem. Soc. 127, 12492-12493

54 Zhang, Z. et al. (2006) Delivery of telomerase reverse transcriptase small interfering RNA in complex with positively charged single-walled carbon nanotubes suppresses tumor growth. Clin. Cancer Res. 12, 4933-4939

55 Firme, C.P., 3rd and Bandaru, P.R. (2010) Toxicity issues in the application of carbon nanotubes to biological systems. Nanomedicine 6, 245-256

56 Zhang, L. et al. (2010) Carbon nanotube uptake and toxicity in the brain. Methods Mol. Biol. 625, 55-65

$57 \mathrm{Wu}$, Y. et al. (2011) A novel magneto-fluorescent nano-bioprobe for cancer cell targeting, imaging and collection. Appl. Biochem. Biotechnol. 163, 813-825

58 Sonavane, G. et al. (2008) Biodistribution of colloidal gold nanoparticles after intravenous administration: effect of particle size. Colloids Surf. B Biointerfaces 66, 274-280

59 Zhao, E. et al. (2012) Surface engineering of gold nanoparticles for in vitro siRNA delivery. Nanoscale 4, 5102-5109

60 Dreaden, E.C. et al. (2011) Beating cancer in multiple ways using nanogold. Chem Soc. Rev. 40, 3391-3404

61 Giljohann, D.A. et al. (2009) Gene regulation with polyvalent siRNA-nanoparticle conjugates. J. Am. Chem. Soc. 131, 2072-2073

62 Davis, M.E. (2009) The first targeted delivery of siRNA in humans via a selfassembling, cyclodextrin polymer-based nanoparticle: from concept to clinic. Mol. Pharm. 6, 659-668

63 Thomas, O. and Lagarce, F. (2013) Lipid nanocapsules: a nanocarrier suitable for scale-up process. J. Drug Del. Sci. Tech. 23, 555-559

64 Deng, Y. et al. (2014) Therapeutic potentials of gene silencing by RNA interference: principles, challenges, and new strategies. Gene 538, 217-227

65 Birmingham, A. et al. (2006) 3' UTR seed matches, but not overall identity, are associated with RNAi off-targets. Nat. Methods 3, 199-204

66 Xue, H.Y. et al. (2014) Nanotoxicity: a key obstacle to clinical translation of siRNAbased nanomedicine. Nanomedicine 9, 295-312

67 Wang, Z. et al. (2011) RNA interference and cancer therapy. Pharm. Res. 28, 2983-2995

68 Castanotto, D. et al. (2007) Combinatorial delivery of small interfering RNAs reduces RNAi efficacy by selective incorporation into RISC. Nucleic Acids Res. 35, $5154-5164$

69 Loscher, W. and Potschka, H. (2005) Role of drug efflux transporters in the brain for drug disposition and treatment of brain diseases. Prog. Neurobiol. 76, 22-76

70 Huse, J.T. and Holland, E.C. (2010) Targeting brain cancer: advances in the molecular pathology of malignant glioma and medulloblastoma. Nat. Rev. Cancer 10, 319-331

71 Tzeng, S.Y. and Green, J.J. (2013) Therapeutic nanomedicine for brain cancer. Ther. Deliv. 4, 687-704

72 Zhang, R. et al. (2014) Concentration rather than dose defines the local brain toxicity of agents that are effectively distributed by convection-enhanced delivery. J. Neurosci. Methods 222, 131-137

73 Bidros, D.S. et al. (2010) Future of convection-enhanced delivery in the treatment of brain tumors. Future Oncol. 6, 117-125

74 Viel, T. et al. (2013) Optimizing glioblastoma temozolomide chemotherapy employing lentiviral-based anti-MGMT shRNA technology. Mol. Ther. 21, 570-579 
75 Han, L. et al. (2010) Combination gene therapy with PTEN and EGFR siRNA suppresses U251 malignant glioma cell growth in vitro and in vivo. Med. Oncol. 27 $843-852$

76 Zhao, Y.Y. et al. (2013) ShRNA-mediated silencing of MDM2 inhibits growth of HepG2 hepatocellular carcinoma cells xenografted in nude mice. Zhonghua Gan Zang Bing Za Zhi 21, 213-217

77 Kato, T. et al. (2010) Efficient delivery of liposome-mediated MGMT-siRNA reinforces the cytotoxity of temozolomide in GBM-initiating cells. Gene Ther. 17, 1363-1371
78 Le Mercier, M. et al. (2008) Knocking down galectin 1 in human hs683 glioblastoma cells impairs both angiogenesis and endoplasmic reticulum stress responses. J. Neuropathol. Exp. Neurol. 67, 456-469

79 Wang, X. et al. (2013) Mutant TP53 enhances the resistance of glioblastoma cells to temozolomide by up-regulating $\mathrm{O}(6)$-methylguanine DNA-methyltransferase. Neurol. Sci. 34, 1421-1428

80 Yang, Y.P. et al. (2012) Inhibition of cancer stem cell-like properties and reduced chemoradioresistance of glioblastoma using microRNA145 with cationic polyurethane-short branch PEI. Biomaterials 33, 1462-1476 